Original Paper

\title{
Early Growth Response 1-Dependent Downregulation of Matrix Metalloproteinase 9 and Mouse Double Minute 2 Attenuates Head and Neck Squamous Cell Carcinoma Metastasis
}

\author{
Jinkyung Kim ${ }^{\mathrm{a}}$ Sung-Min Kang ${ }^{\mathrm{a}}$ Su Young Oha Soo Hyun Kang ${ }^{\mathrm{a}}$ \\ Inhan Lee ${ }^{c}$ Jae Chan Hwang ${ }^{c}$ Heon-Jin Lee ${ }^{a}$ So-Young Choi ${ }^{b}$ \\ Su-Hyung Honga \\ aDepartment of Microbiology and Immunology, School of Dentistry, Kyungpook National University, \\ Daegu, bepartment of Oral and Maxillofacial Surgery, School of Dentistry, Kyungpook National \\ University, Daegu, South Korea, 'MIRCORE Research Org., Ann Arbor, MI, USA
}

\section{Key Words}

Head and neck squamous cell carcinoma $•$ Early growth response 1 - The Cancer Genome Atlas

- Matrix metalloproteinases $•$ Mouse double minute 2 homolog

\begin{abstract}
Background/Aims: The functional relevance of early growth response-1 (EGR1) on cancer invasion remains controversial. The effect of EGR1 on the expression of MMP9, which is important for HNSCC invasion, is still disputed. There is no previous data showing the effect of EGR1 on mouse double minute 2 (MDM2), an enhancer of matrix metalloproteinase 9 (MMP9) expression. Our aim is to clarify the negative correlation between EGR1 expression and head and neck squamous cell carcinoma (HNSCC) metastasis. Methods: EGR1 mRNA and protein expressions were compared in normal and HNSCC tissues using The Cancer Genome Atlas (TCGA) dataset analysis or immunohistochemistry (IHC), respectively. In vitro cell invasion was evaluated Matrigel invasion assay. EGR1-dependent inhibition of MDM2 transcription was assessed by promoter-luciferase assay and chromatin immunoprecipitation (ChIP). Results: TCGA data showed that EGR1 mRNA levels are significantly higher in normal oral tissues as compared with HNSCC tumor tissues (adjusted $\mathrm{P}=1.64 \times 10^{-16}$ ). In addition, nonmetastatic HNSCC tissues showed significantly higher EGR1 mRNA levels as compared with metastatic tissues (adjusted $P=0.023$ ). IHC analysis showed that primary tumor tissues expressed significantly higher levels of nuclear EGR1 compared with paired metastatic lymph node tissues $(P<0.05)$. EGR1 overexpression downregulated MMP9 and MDM2 protein expression.

J. Kim and S. Kang contributed equally to this work.

\begin{tabular}{ll}
\hline $\begin{array}{l}\text { Su-Hyung Hong } \\
\text { and So-Young Choi }\end{array}$ & Department of Microbiology and Immunology, Department of Oral and Maxiollofacial Surgery, \\
& Kyungpook National University, 2177 Dalgubeol-daero, Jung-gu, Daegu 700-412 (South Korea) \\
& E-Mail hongsu@knu.ac.kr; dentalchoi@knu.ac.kr
\end{tabular}
\end{abstract}


Consistent with these observations, TCGA data analysis found significantly fewer metastatic patients among a subgroup of population presenting higher EGR1 expressions with lower MMP9 and/or MDM2. Conclusion: Our data suggests that EGR1 prevents HNSCC metastasis through downregulation of MMP9 and MDM2. EGR1 might be a potential candidate to attenuate HNSCC metastasis.

\section{Introduction}

Cancer metastases, rather than primary tumors, are responsible for most cancer deaths $[1,2]$. The recurrence rate in patients treated for HNSCC remains unacceptably high, at up to $50 \%$ within the first two years [3]. Understanding the molecular mechanisms that mediate HNSCC invasion and metastasis might enable to prevent HNSCC recurrence. Early growth response-1 (EGR1), a zinc finger transcription factor, regulates a remarkable spectrum of cellular responses, including survival, apoptosis, differentiation, and transformation [4, 5]. EGR1 is either a tumor-suppressor or tumor-promoter, depending on the cell type and environment. EGR1 exhibits a prominent tumor suppressor function in lung cancer and glioma $[6,7]$. On the other hand, EGR1 promotes the progression of prostate cancer [8]. In addition, abnormal expression of EGR1 in gastric cancer is known to be associated with tumor metastasis [9]. However, the molecular mechanism by which EGR1 regulates cancer metastasis is not well-understood. It is well known that MMP2 and MMP9 is important for HNSCC invasion. Previous study showed that EGR1 inhibits MMP2 expression by direct promoter binding in human breast cancer cell [10]. EGR1 has been reported to promote MMP9 expression in HeLa cells [11], but to inhibit its expression in lymphoma [12]. It has been known that MDM2 promotes metastasis of breast cancer by upregulating MMP9 [13]. Furthermore, aberrant MMP9-MDM2 expression is associated with lung cancer progression [14]. Therefore, in this study, we evaluated the relationship between EGR1 expression and HNSCC metastasis in association with MMP9 and MDM2.

EGR1 protein levels in HNSCC tumor tissues, especially primary and paired metastatic tumors, remain largely unknown. Therefore, we used immunohistochemistry (IHC) to compare EGR1 protein expression patterns in HNSCC tumor tissues, including non-metastatic primary tumors, primary and paired metastatic tumor tissues. Well-defined multiple types of The Cancer Genome Atlas (TCGA) data from a large number of patients can provide unique insight into differential expression of specific genes. We used HNSCC datasets from TCGA to examine the differential expression of EGR1 and MMP9/MDM2 in clinical samples. Considering the diverse nature of metastasis, we also used unsupervised hierarchical clustering to identify a subgroup of patients who present upregulated EGR1 expression and downregulated genes. Our data suggest that EGR1 may prevent HNSCC metastasis through downregulation of MMP9 and/or MDM2.

\section{Materials and Methods}

\section{Chemicals and reagents}

DMEM, RPMI medium, FBS, and antibiotics were obtained from Gibco (Grand Island, NY, USA). Rabbit anti-EGR1 antibody was purchased from Cell Signaling Technology (Danvers, MA, USA). Rabbit anti-MMP2, anti-MMP9, and mouse anti-MDM2 antibodies were purchased from Abcam (Cambridge, MA, USA). Anti$\beta$-actin antibody and secondary antibodies were acquired from Santa Cruz Biotechnology Inc. (Dallas, TX, USA). Matrigel matrix for invasion assay was purchased from Corning (Bedford, MA. USA). Specific MMP2/9 inhibitor (CAS 193807-60-2) and Phorbol-12-Myristate-13-Acetate (PMA) were purchased from Merck Millipore. All other reagents were obtained from standard commercial sources. 


\section{Cellular Physiology Cell Physiol Biochem 2018;50:1869-1881

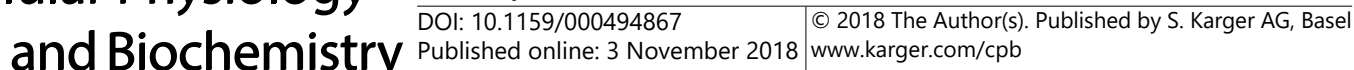 \\ Kim et al.: EGR1 Attenuates HNSCC Metastasis}

\section{TCGA RNAseq data analysis}

We downloaded all available clinical supplement data for TCGA-HNSCC project participants from the NCI GDC Data Portal. Using the clinical supplement data and in-house software, we classified the patients as either metastatic or non-metastatic based on their AJCC clinical N score, where a score of N0 was considered non-metastatic and a score of N1-N3 was considered metastatic. Patients with a clinical N score of NX were removed from our study.

We downloaded the RNA-seq gene expression quantification count data (HTSeq) of all HNSCC patient tissues from GDC Data Portal. We used DESeq2 [15] for differential gene expression analysis to compare 1) normal $(n=43)$ and tumor tissues $(n=500)$ from HNSCC patients, and 2) tumor tissues between meta (n $=245)$ and non-meta $(n=238)$ groups. In addition to the HTSeq count data, we also downloaded FPKMUQ (Fragments Per Kilobase of transcript per Million mapped reads upper quartile) normalized RNA-seq data of the same samples for expression value comparison across all samples. Box plots of EGR1 FPKM-UQ expressions of normal and tumor tissues were generated using R. Line plots of EGR1 FPKM-UQ expressions for both the metastatic group and non-metastatic group were generated after sorting the values from lowest to highest expression within each group in order to easily visualize the difference in EGR1 expression between the two groups. Log2 transformed FPKM-UQ data were used to perform unsupervised hierarchical clustering using the $\mathrm{R}$ package heatmap [https://CRAN.R-project.org/package=pheatmap] with the clustering distance measured using Pearson correlation.

\section{Immunohistochemical analysis}

Paraffin-embedded tissue blocks from each of the 10 non-metastatic HNSCC tissues, metastatic primary, and paired metastatic lymph node tissues were obtained from patients with HNSCC who underwent tumor resection for oral cancer treatment from 2010-2015 at the Kyungpook National University Hospital. Informed consent was obtained from all patients before the collection of specimens. The study was approved by the Ethics Committee of the Kyungpook National University Hospital. The patients' information is as follows: 10 metastatic and 10 non-metastatic patients with average ages of $57.7 \pm 12.1$ and $61.2 \pm$ 7.8 years, respectively; each group comprised eight males and two females. After dewaxing, sections (5 $\mu \mathrm{m}$ ) were blocked for $5 \mathrm{~min}$, then incubated for $2 \mathrm{~h}$ at room temperature with EGR1 antibody (1:50). IHC staining was performed using the UltraTek HRP Anti-Polyvalent kit (ScyTek Laboratories, Logan, UT, USA); the chromogen used was 3, 3'-diaminobenzidine (Dako, Carpinteria, CA, USA). Nuclei were counterstained with hematoxylin. Two experienced researchers who were blinded to the origin of the sections evaluated the immunoreactivity independently. EGR1-positive cells were counted in 100 cells of each sample: 10 representative fields at $400 \times$ magnification were selected and the cells were counted. Thus, by selecting 10 random areas that were not contiguous with each other, possible errors in recounting the same cell were minimized.

\section{Cell culture}

FaDU and UMSCC1 were obtained from the American Type Culture Collection (Manassas, VA, USA) and Merck KGaA (Darmstadt, Germany), respectively, and maintained in DMEM or RPMI medium containing $10 \%$ FBS and $1 \%$ penicillin/streptomycin solution. Cells were grown at $37^{\circ} \mathrm{C}$ in a $5 \% \mathrm{CO}_{2}$ humidified atmosphere.

\section{EGR1 overexpression vector transfection}

The full-length EGR1 cDNA was generated by PCR from a human cDNA clone and the amplified products were cloned into the pCDNA3.1 myc-HisA vector [16]. Cells were transiently transfected with a control pCDNA3.1 vector or EGR1-overexpression vector using an electroporation system (Bio-Rad Laboratories, Inc., Hercules, CA, USA) or Lipofectamine ${ }^{\mathrm{TM}} 2000$ (Thermo Fisher Scientific USA).

Quantitative real-time PCR ( $q P C R$ )

Total RNA extraction, cDNA synthesis, and gene expression normalization were carried out according to the standard protocols [16]. The following primers were used for qPCR: EGR1, forward, 5'-CAGGAGTGATGAACGCAAGA-3', reverse, 5'-GGGATGGGTAGGAAGAGAGG-3'; MMP2, forward, 5'-AGCTGCAACCTGTTTGTGCTG-3', reverse, 5' -CGCATGGTCTCGATGGTATTCT-3'; MMP9 forward, 5'-ACGACGTCTTCCAGTACCGAGA, reverse, 5'-TAGGTCACGTAGCCCACTTGGT; MDM2 forward, CCCAAGACAAAGAAGAGAGTGTGG, reverse, CTGGGCAGGGCTTATTCCTTTTCT; and GAPDH, forward, 


\section{Cellular Physiology Cell Physiol Biochem 2018;50:1869-1881 \begin{tabular}{l|l|l} 
and Biochemistry Published online: 3 November 2018 & $\begin{array}{l}\text { ○ } 2018 \text { The Author(s). Published by S. Karger AG, Basel } \\
\text { www.karger.com/cpb }\end{array}$ \\
\hline
\end{tabular} \\ Kim et al.: EGR1 Attenuates HNSCC Metastasis}

5'-AGATCATCAGCAATGCCTCCTG-3', reverse, 5'-ATGGCATGGACTGTGGTCATG-3'. Gene expression was normalized to the expression of the housekeeping gene GAPDH. qPCR was carried out using an ABI 7500 real-time PCR system (Applied Biosystems).

\section{Western blotting analysis}

Protein extraction and determination of concentration were carried out according to the standard protocols [16]. Aliquots of the protein samples $(30-40 \mu \mathrm{g})$ were electrophoresed on 8-15\% SDS-PAGE gels. The proteins were then transferred to nitrocellulose membranes, blocked in $5 \%$ skim milk for $2 \mathrm{~h}$ at room temperature, and subsequently incubated with primary antibodies overnight at $4^{\circ} \mathrm{C}$. HRP-conjugated secondary antibodies were applied at 1:5000 dilutions for $1 \mathrm{~h}$ at room temperature, after which the blot was washed three times in TBST (TBS and $0.1 \%$ Tween 20). $\beta$-Actin was used as a loading control. The proteins were detected using ECL detection reagents and the relative intensities of the bands were analyzed using Image software.

\section{2-D and 3-D Matrigel invasion assay}

First, in vitro 2-D cell invasion was evaluated using Matrigel-coated, $8.0 \mu \mathrm{m}$ filter chambers (BD Biosciences, San Jose, CA, USA). Vector-transfected FaDU cells were added to each Matrigel-coated Transwell. After culture for $48 \mathrm{~h}$, cell invasion was carried out according to the standard protocols [16]. The invasion index was calculated as the fold change of invaded cells in the experimental groups compared with that in the control group.

3-D spheroids' Matrigel invasion was evaluated in a 96-well plate format with one spheroid per well [17]. FaDU cells were seeded in a 96-well U-bottom ultra-low attachment plate (5000 cells per well), then cultured for 4 days to form spheroids (approximately $700 \mu \mathrm{m}$ in diameter). Lipofectamine 2000 and transfection vector were added to the spheroids in a 96-well plate. After $8 \mathrm{~h}, 50 \mu \mathrm{l}$ Matrigel matrix was added directly to each well $(100 \mu \mathrm{l}$ medium) to provide a semi-solid matrix into which tumor cells invade from the spheroid body. After Matrigel was solidified, $100 \mu \mathrm{l}$ of the medium was added. Matrigel invasion was monitored over a period of 10 days by phase-contrast microscopy ( $5 \mathrm{X}$ magnification), and quantified by measuring the average length of tube-like structures from the surface of each spheroid.

\section{Promoter-luciferase assay}

A previous study showed that MDM2 promotes cancer invasion and metastasis in breast carcinoma by inducing MMP9 [13]. Interestingly, Sp1 binds to the promoter sequence of MDM2 genes for upregulation [18], and that EGR1 competes with Sp1 for the same promoter sequences [19]. Therefore, we performed promoter-luciferase analysis in control vector or EGR1 overexpression vector-transfected FaDU cells to evaluate whether EGR1 transregulates MDM2 gene expression. The MDM2 (-733 to -230) promoter fragments were synthesized from human genomic DNA (Takara). The amplified PCR products were cloned into the pGL4.70 vector (Promega, Madison, WI, USA) upstream of the Renilla luciferase coding sequence. For the reporter assay, pGL4.70 with MDM2 DNA, and the pcDNA3.1 control vector or pcDNA3.1-EGR1 overexpression vector were co-transfected via electroporation. The pGL3-basic firefly (Photinus pyralis) luciferase plasmid was co-transfected as a normalization control. The MDM2 mutant promoter constructs were synthesized using the combined overlap extension PCR method [20]. All assays were performed $24 \mathrm{~h}$ after transfection using the dual luciferase assay (Promega), according to the manufacturer's protocol.

\section{Chromatin immunoprecipitation (ChIP) analysis}

ChIP was performed using a rabbit anti-EGR1 antibody or normal rabbit IgG during overnight at 4 ${ }^{\circ} \mathrm{C}$. qPCR was performed using $3 \mu \mathrm{l}$ of immunoprecipitated chromatin or input chromatin, using primers flanking the EGR1 binding sequences of MDM2 promoters ( -460 to -455 and -391 to -386 ).

\section{Statistical analysis}

Enrichment testing of metastatic tumor in a subgroup of TCGA data was done by the hypergeometric distribution. Significant variation analysis was used to calculate the parametric: a two-tailed, nonpaired $t$-test, analysis of variance, and the non-parametric Mann-Whitney $U$-test were used to compare two populations form TCGA dataset. All other analyses were performed using Origin 8.0 (OriginLab, Northampton, MA, USA), and P $\leq 0.05$ was considered statistically significant. 


\section{Cellular Physiology Cell Physiol Biochem 2018;50:1869-1881 \begin{tabular}{ll|l} 
and Biochemistry & $\begin{array}{l}\text { DOI: 10.1159/000494867 } \\
\text { Published online: 3 November } 2018\end{array}$ & $\begin{array}{l}\text { (c) } 2018 \text { The Author(s). Published by S. Karger AG, Basel } \\
\text { www.karger.com/cpb }\end{array}$
\end{tabular}

\section{Results}

EGR1 expression was higher in nonmetastatic tissues of HNSCC patients

We first analyzed TCGA data to compare EGR1 mRNA expressions in non-tumor oral tissues and HNSCC tumor tissues. As shown in Fig. 1A, EGR1 mRNA expression is significantly higher in normal tissues than those in tumor tissues of HNSCC patients (Benjamini-Hochberg adjusted $\left.\mathrm{P}=1.64 \times 10^{-16}\right)$. Next, we identified 245 patients with cancers metastasized to lymph nodes (metastatic group) and 238 patients without metastasis (nonmetastatic group) from the clinical data of HNSCC patients. Nonmeta group refers to a group with no cancer metastasis, that is, only with a primary tumor. Raw counts of RNAseq data of tumors between these two groups compared using DESeq2 package revealed significantly downregulated EGR1 expressions in the tumors of metastatic cancers (Benjamini-Hoschberg adjusted P = 0.023). To provide all EGR1 expression values of tumor samples in both groups, the sorted upper quartile normalized FPKM values of EGR1 in nonmeta and meta groups are shown in Fig. 1B.

EGR1 protein-positive cells between nonmetastatic and metastatic primary tumor tissues of HNSCC patients' were compared (Fig. 2A). There was no significant difference in EGR1 protein expression between these two groups (P > 0.05). We then compared EGR1 expression in metastatic primary and paired lymph node metastatic tumors. The average number of EGR1-positive tumor cells per patient tissue is displayed in Fig. 2B. The ratio of EGR1-positive cells between metastatic primary tumors versus paired lymph node tumors was calculated. As shown in Fig. 2C, primary tumors showed significantly higher EGR1 protein levels compared with those in the paired lymph node tumors. Two representative immunohistochemical image sets of metastatic primary and paired lymph node tumor tissues are shown in Fig. 2D.

(A)

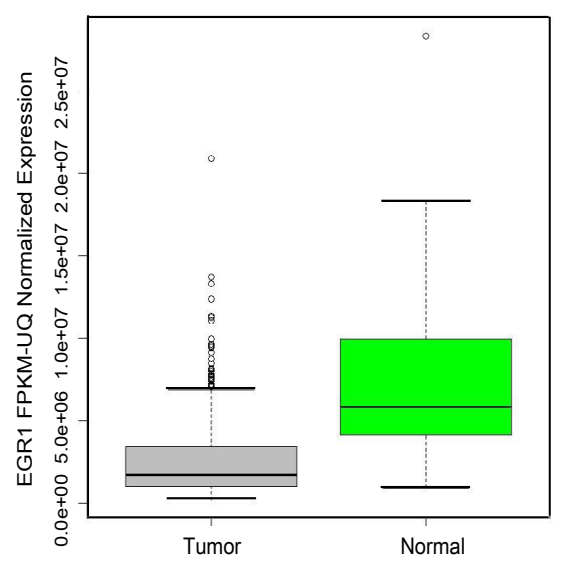

(B)

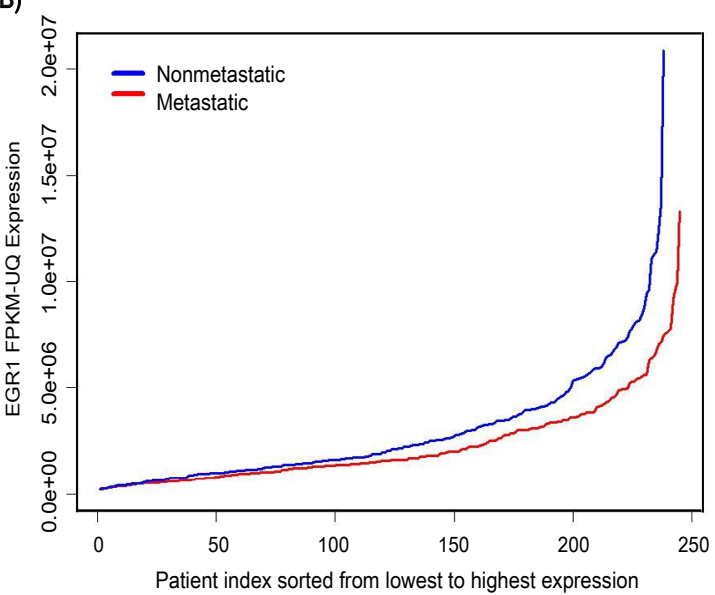

Fig. 1. EGR1 mRNA expressions in normal oral and HNSCC patients' tissues. (A) Box plots of expression data of normal $(n=43)$ and tumor $(n=500)$ tissues. The DESeq2 calculation for adjusted $p$-value was performed with Benjamini-Hochberg method (adjusted $\mathrm{P}=1.64 \times 10^{-16}$ ). (B) EGR1 mRNA expressions of individual patients' tumor for those who have specific AJCC clinical $N$ values $(n=483)$. DESeq2 was used for differential gene expression analysis between metastatic $(n=245)$ and non-metastatic $(n=238)$ groups. Plots of EGR1 FPKM-UQ (Fragments Per Kilobase of transcript per Million mapped reads upper quartile) expressions for both the metastatic (red line) and non-metastatic (blue line) group were generated after sorting the values from lowest to highest expression within each group (adjusted $\mathrm{P}=0.023$ ). 


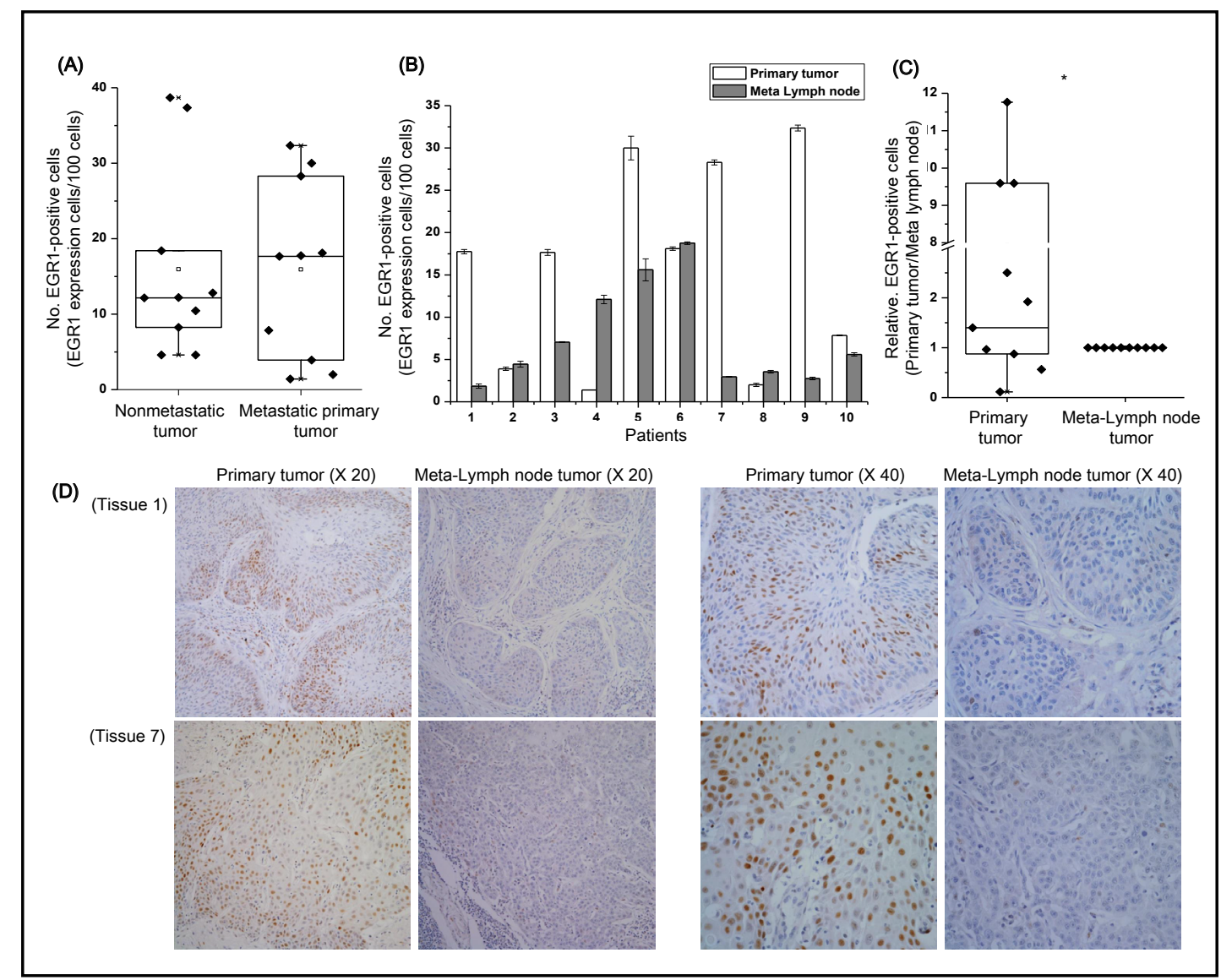

Fig. 2. EGR1 protein expressions in HNSCC patients' tissues. (A) EGR1 protein expression was compared in non-metastatic tumors and metastatic primary tumors. (B) EGR1 expression in primary and paired metastatic lymph node tumor tissues was compared. (C) To compare EGR1-positive tumor cells between primary and paired metastatic tumors, the relative EGR1 expression was calculated as the number of EGR1positive cells in metastatic tumors compared with that of primary tumors $\left({ }^{*} \mathrm{P}<0.05\right)$. The boxes show the upper $75 \%$ and lower $25 \%$ quartiles of the measurements with respect to the median value (horizontal line in each box). Each dot represents an individual patient's data, while a small open rectangle represents the mean value. Each bar represents the standard deviation. Representative images are shown in (D)

\section{EGR1 upregulation inhibited transwell Matrigel invasion of HNSCC cells}

Because the endogenous EGR1 expression was relatively low in the HNSCC cells, EGR1 overexpression vector was used to investigate the effect of EGR1 on HNSCC cell invasion. EGR1 overexpression was confirmed by qPCR and western blot analysis (Fig. 3A). Protein expression of MMP2, MMP9, and MDM2 were reduced remarkably in EGR1-overexpressing cell (Fig. 3B). To further confirm whether MMP2 or MMP9 affects MDM2 expression, specific MMP2/9 inhibitor (final concentration of $30 \mathrm{nM}$ ) was treated for $24 \mathrm{~h}$, followed by MDM2 protein expression analysis. As shown in Fig. 3C, there was no remarkable change of MDM2 protein expression with MMP2/9 inhibitor, suggesting that decreased MDM2 protein expression in EGR1-overexpressing cell would be due to EGR1. Furthermore, the effect of PMA, a strong inducer of MMP9, on EGR1-dependent MMP9 downregulation was evaluated at FaDU cell. As shown in Fig. 3D, MMP9 protein expression was increased with PMA treatment for $24 \mathrm{~h}(50 \mathrm{ng} / \mathrm{ml})$ in control vector-transfected FaDU cell. However, in EGR1 overexpressiontransfected cell, PMA-dependent MMP9 upregulation was disappeared. Microscopic analysis of the Matrigel invasion in HNSCC cells showed that in vitro cell invasion was decreased significantly by transient EGR1 overexpression in HNSCC cells $(\mathrm{P}<0.001)$ (Fig. 3E). 
(A) FaDU

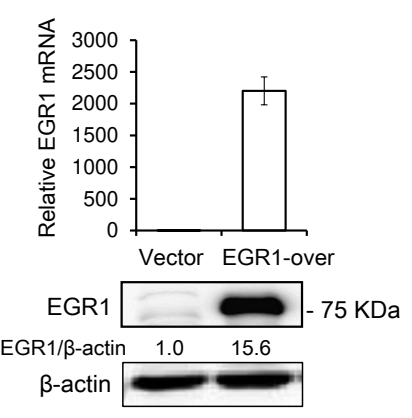

(E)

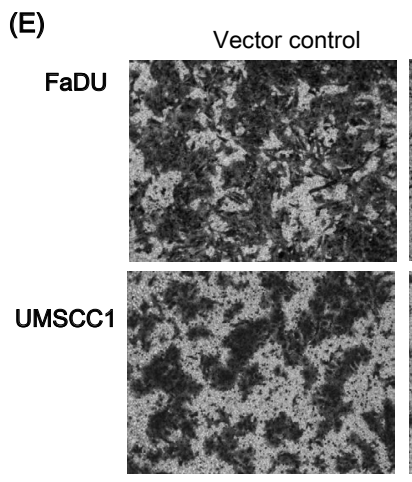

(B) FaDU
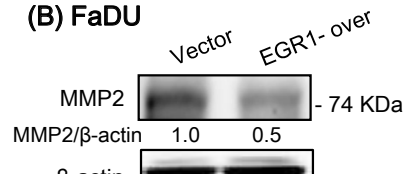

$\beta$-actin

MMP

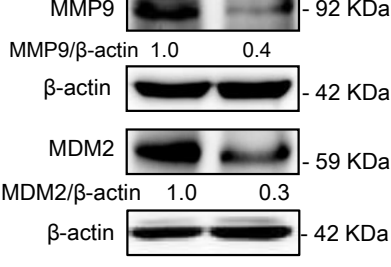

EGR1-over

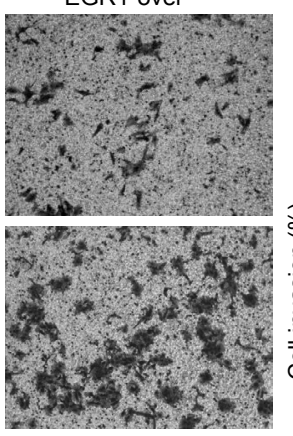

(C) FaDU

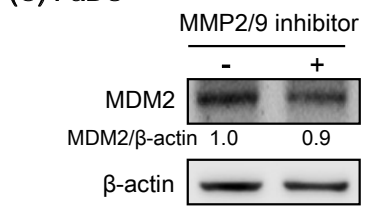

(D)

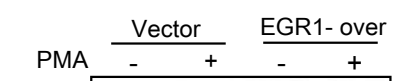

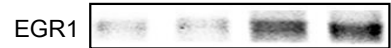
EGR1/ $\beta$-actin $1.0 \quad 1.1 \quad 8.5 \quad 9.2$

$\beta$-actin $\approx$ m

MMP9

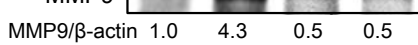

$\beta$-actin $=0$

FaDU

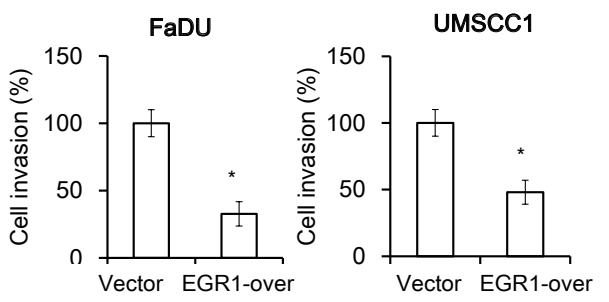

Fig. 3. Effects of EGR1 overexpression on in vitro Matrigel invasion of HNSCC cells. (A) pcDNA3.1 EGR1 overexpression vector ("EGR1-over") was transfected for $48 \mathrm{~h}$ in FaDU cell and EGR1 mRNA and protein abundance was evaluated by qPCR and western blot analysis. (B) MMP2, MMP9, and MDM2 protein levels were compared at the same condition. (C) Specific MMP2/9 inhibitor was treated for $24 \mathrm{~h}$ in FaDU cell (final concentration of $30 \mathrm{nM}$ ), and then MDM2 protein expression was analyzed. (D) FaDU cell was transfected with EGR1 overexpression vector for $16 \mathrm{~h}$, followed by PMA treatment for $24 \mathrm{~h}$ more (final concentration of $50 \mathrm{ng} / \mathrm{ml}$ ). EGR1 and MMP9 protein expression was analysed with western blotting. (E) A Transwell Matrigel invasion assay was performed to analyze the effect of EGR1 overexpression on FaDU and UMSCC1 cells invasion. The cell invasion index was calculated as the difference between invaded cells in the EGR1 overexpression vector-transfected group compared with those in the control pcDNA vector-transfected group $\left({ }^{*} \mathrm{P}<0.001\right)$. The results shown are from two or three independent experiments, with each bar representing the standard deviation.

\section{EGR1 upregulation inhibited HNSCC cell spheroids' Matrigel invasion}

Fig. 4A shows the upregulation of EGR1 mRNA with EGR1 overexpression vector transfection for 13 days in FaDU spheroids. EGR1 protein expression was evaluated at day 7 after transfection. mRNA expression of MMP2, MMP9, and MDM2 in FaDU spheroids was decreased by EGR1 overexpression (Fig. 4B). UMSCC1 spheroids showed the same expression pattern as that of FaDU (Fig. 4C). Fig. 4D shows the representative image of Matrigel invasion of FaDU spheroids transfected with vector. There was no remarkable invasion for 4 days following transfection. Quantification of invasion on the basis of 4-day invasion of control vector-transfected spheroids is shown at Fig. 4E. After 7 days, there was a significant decrease of Matrigel invasion in EGR overexpression vector-transfected spheroids as compared with control vector-transfected ones $\left({ }^{*} \mathrm{P}<0.005,{ }^{* *} \mathrm{P}<0.0005\right)$. 


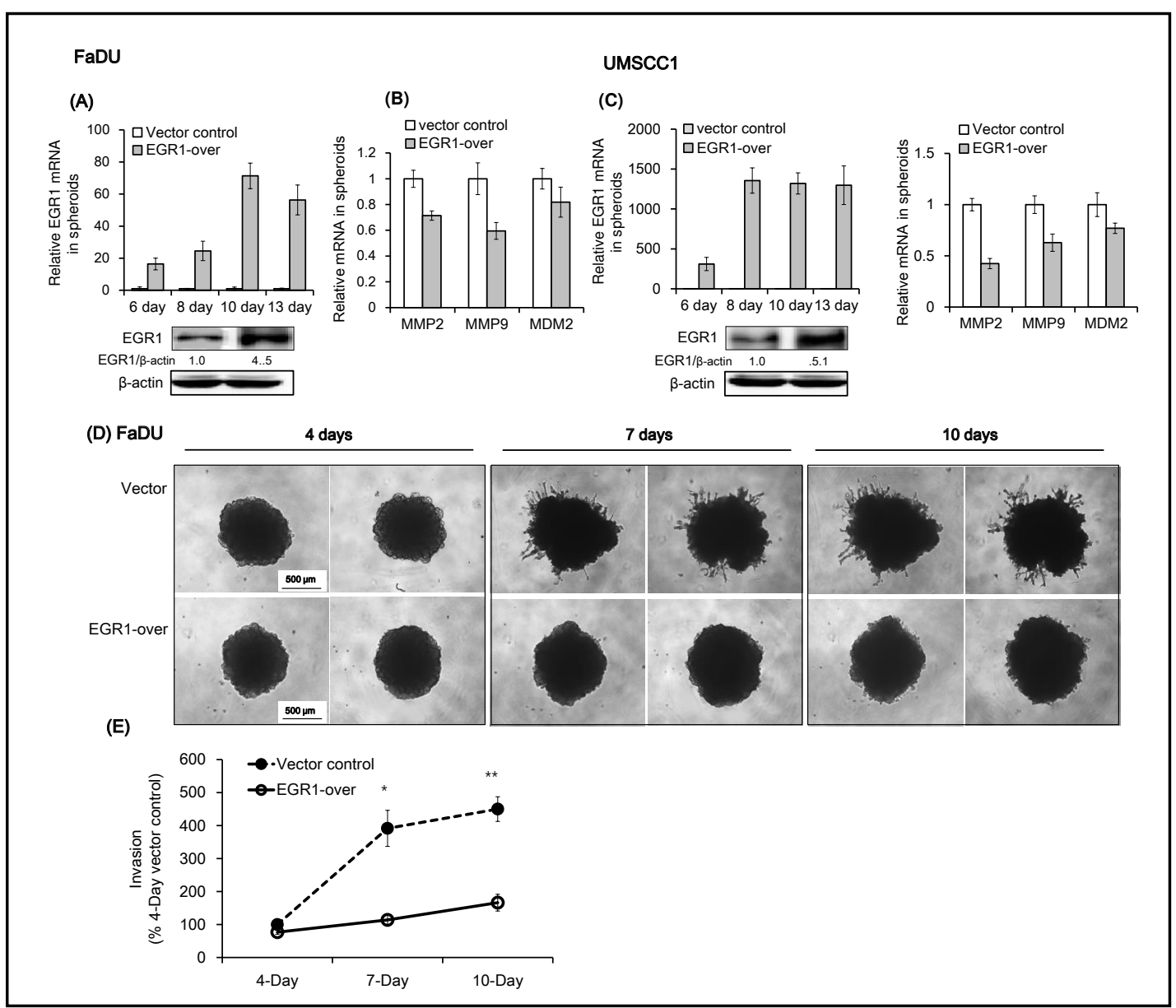

Fig. 4. Effects of EGR1 on 3-D Matrigel invasion. FaDU cells were seeded in a 96-well U-bottom plate (5000 cells per well), then cultured for 4 days to form one spheroid per well (approximately $700 \mu \mathrm{m}$ in diameter). (A) EGR1 overexpression vector or control vector was transfected to FaDU spheroids with Lipofectamine 2000, and EGR1 mRNA expression was evaluated by qPCR for 13 days. EGR1 protein expression from spheroids was analyzed after 7 days of transfection. (B) mRNA expression of MMP2, MMP9, and MDM2 in FaDU spheroids were investigated after 7 days of transfection. (C) mRNA and/or protein expression of EGR1, MMP2, MMP9, and MDM2 in UMSCC1 spheroids were investigated at the same condition. (D) After $8 \mathrm{~h}$ of transfection, the spheroids were centrifuged to spin down, after which $50 \mu$ Matrigel matrix was added to $100 \mu \mathrm{l}$ medium in 96-well plate. 3-D Matrigel invasion was observed for 10 days by phase-contrast microscopy (5x magnification). (E) Matrigel invasion was quantified by the average length of tube-like structures from the surface of each spheroid $\left({ }^{*} \mathrm{P}<0.005,{ }^{*} \mathrm{P}<0.0005\right)$. All experiments were repeated two or three times, with each bar representing the standard deviation.

\section{EGR1 downregulated MDM2 mRNA expression by direct promoter binding}

Fig. $5 \mathrm{~A}$ shows a schematic representation of the promoter regions containing the consensus EGR1-binding sites that were searched using the AliBaba 2.1 program. Promoter sequences with site-directed mutations are shown in red. As shown in Fig. 5B, MDM2 promoter-luciferase activity decreased significantly in EGR1-overexpressing cells ${ }^{*} \mathrm{P}$ $<0.00005)$. However, site-directed mutations on MDM2 promoter abolished the effect of EGR1 overexpression (Fig. 5C). In addition, ChIP analysis showed that binding of EGR1 to the consensus sequences in MDM2 promoter (Fig. 5D) increased remarkably in the EGR1overexpressing cells (Fig. 5E) $\left.{ }^{* *} \mathrm{P}<0.005\right)$. Fig. $5 \mathrm{~F}$ shows a schematic representation of the regulation of cell migration by EGR1. 


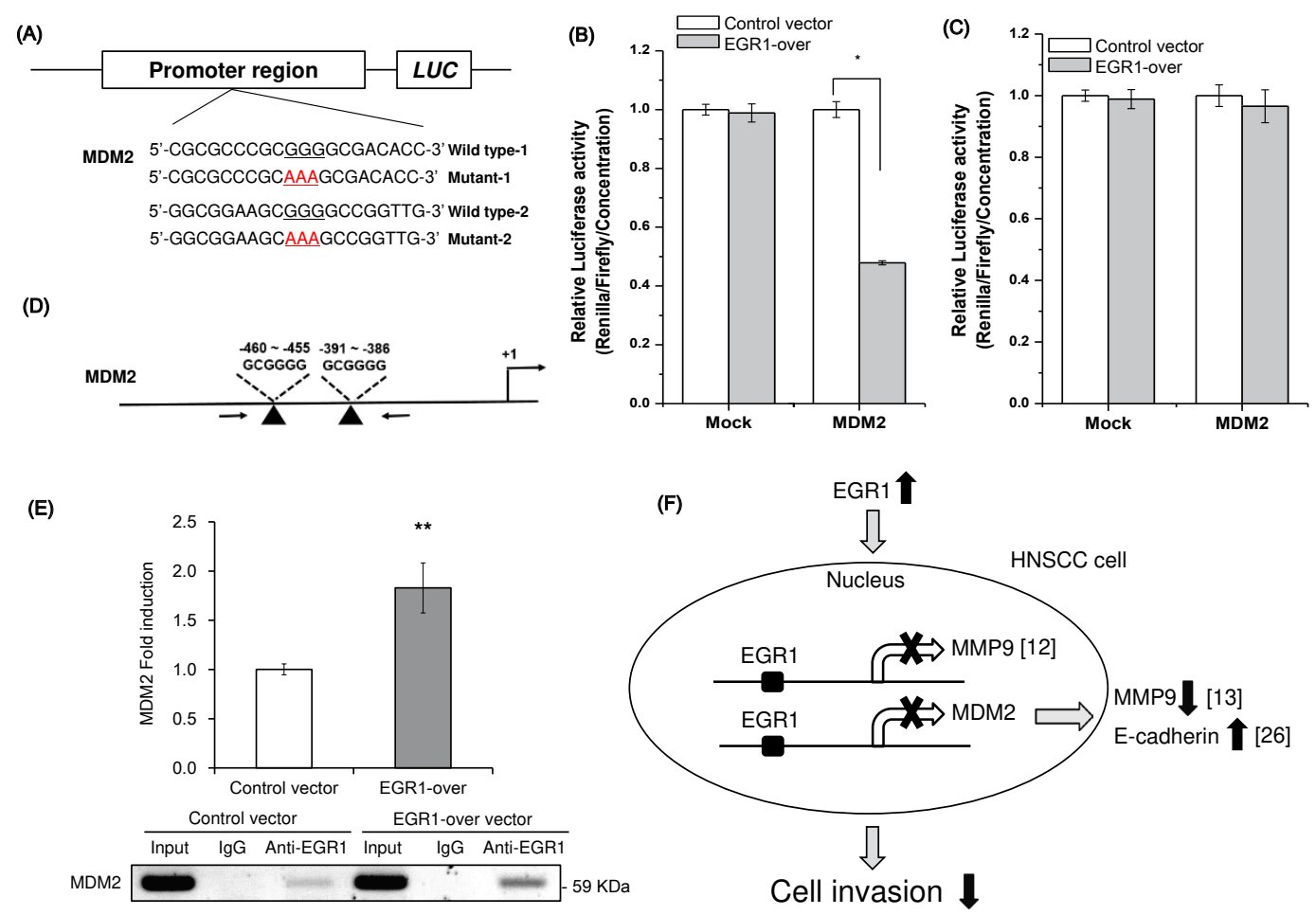

Fig. 5. EGR1-dependent downregulation of MDM2. (A) Diagram of luciferase reporter gene construct of MDM2. The mutated nucleotides in each fragment are shown in red. (B) For the luciferase reporter assay of MDM2 (-733 to -230), wild type promoter fragments were synthesized and cloned into the pGL4.70 vector. After $24 \mathrm{~h}$ of vector transfection, the dual luciferase assay was performed $(* \mathrm{P}<0.00005)$. (C) Luciferase reporter assay with mutant promoter fragments were performed at the same condition. (D) Schematic presentation of the promoter regions of the MDM2 genes showing the consensus EGR1 binding sites in ChIP analysis (black arrowheads). (E) Chromatin of FaDU cells was immunoprecipitated using EGR1 antibody and the resulting immunoprecipitates were analyzed by qPCR to detect the consensus sequence of the promoters of each gene $\left({ }^{* *} \mathrm{P}<0.05\right)$. PCR products were compared by gel electrophoresis. Input and IgG were used as positive and negative controls, respectively. All experiments were performed in triplicate and each bar represents the standard deviation. (F) Flow scheme of EGR1-dependent inhibition of HNSCC cell invasion.

Subgroup of high EGR1 and low MMP9/MDM2 presented more nonmetastatic cancer in the TCGA data

To discern EGR1 function preventing metastasis that we found in the in vitro experiments, we used unsupervised hierarchical clustering of EGR1, MMP9, and MDM2 expressions for all patient tumors from TCGA data. As shown in Fig. 6A, a large subgroup clustered together (113 patients, $23 \%$ of total) presents higher EGR1 and lower MMP9 and/or MDM2 expressions. When we checked the metastatic status of this subgroup, 50 patients were metastatic, while 63 were non-metastatic, presenting a significantly higher number of non-metastatic patients (hypergeometric distribution $\mathrm{P}=0.025$ ). Fig. $6 \mathrm{~B}$ shows the distribution of meta-nonmeta patients according to high EGR1 and low MMP9/MDM2 expression in the heatmap. 


\section{Cellular Physiology Cell Physiol Biochem 2018;50:1869-1881 and Biochemistry \begin{tabular}{l|l} 
DOl: $10.1159 / 000494867$ \\
Published online: 3 November 2018
\end{tabular} $\begin{aligned} & \text { O } 2018 \text { The Author(s). Published by S. Karger AG, Basel } \\
& \text { www.karger.com/cpb }\end{aligned}$

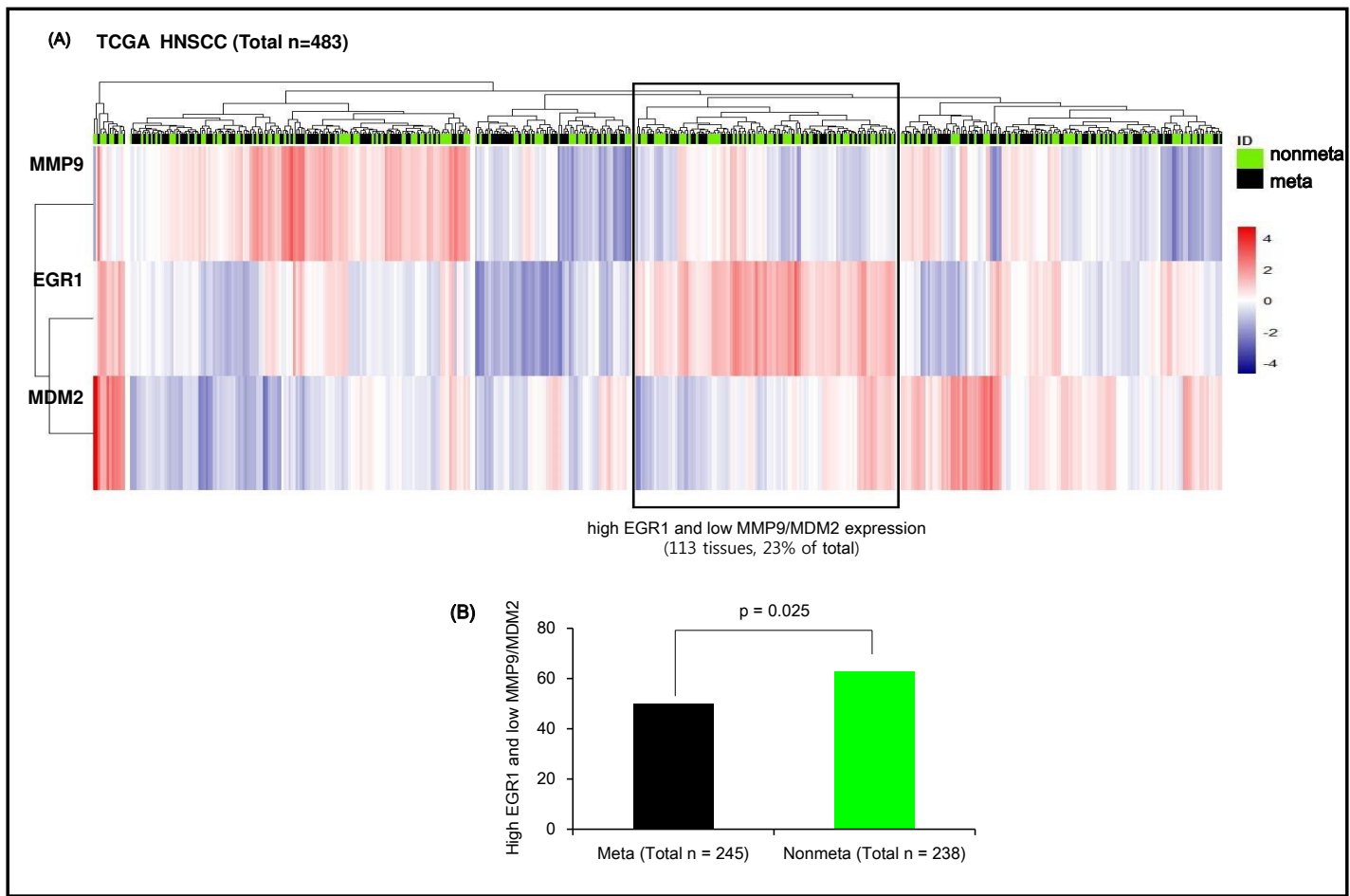

Fig. 6. Heatmap representation of the relative expression levels of EGR1, MMP9, and MDM2 in the TCGA population. (A) Unsupervised hierarchical clustering with distance measured in Pearson correlation was performed on log2 transformed FPKM-UQ normalized expression of EGR1, MMP9, and MDM2 for all patients. Tumors from metastatic and non-metastatic patients were annotated with black and green bars, respectively. A subgroup with a black box among five displays high EGR1 expression and low MMP9/MDM2 expression. (B) The distribution of meta-nonmeta patients according to high EGR1 and low MMP9/MDM2 expression in the heatmap is shown in a bar graph $(\mathrm{P}=0.025)$.

\section{Discussion}

The effect of EGR1 on cancer proliferation and metastasis is still controversial. To evaluate the functional relevance of EGR1 in oral cancer, we first compared EGR1 mRNA level in normal and HNSCC tumor tissues from TCGA datasets. EGR1 mRNA level is significantly higher in normal tissues. Furthermore, non-metastatic HNSCC tissues showed significantly higher EGR1 mRNA level as compared with metastatic HNSCC tissues. There has been no previous IHC data representing EGR1 protein levels in HNSCC tumor tissues. In the present study, there was no significant difference in EGR1 protein levels between non-metastatic tumors and metastatic primary tumors from HNSCC patients. However, in metastatic tumors, nuclear EGR1 expression was significantly higher in primary tumors compared with that in paired metastatic lymph node tumors. Previous study showed that, in colorectal cancers, EGR1 protein expression was higher in the cytoplasm of metastatic lymph node tissues when compared to that in non-metastatic lymph node tissues [21]. It has already been shown that the effects of nuclear EGR1 and cytoplasmic EGR1 on cancer progression are incompatible [22]. The discrepancy of cellular localization of EGR1 protein in HNSCC and in colorectal tumors may explain contradictory effects of EGR1 on each cancer progression.

Recently, more sophisticated screening assays by 3-D cell culture for anti-invasion drugs have been developed to mimic the in vivo cancer microenvironment [23]. The 3-D invasion assay of Matrigel-embedded spheroids provides a rapid invasion system using a highly reproducible, standardized method [24] in a 96-well plate format with one spheroid per well. Interestingly, EGR1 overexpression decreased invasion of Matrigel-embedded spheroids for 10 days. Transient EGR1 overexpression of spheroid cells was achieved with 


\section{Cellular Physiology Cell Physiol Biochem 2018;50:1869-1881 \begin{tabular}{ll|l} 
and Biochemistry & $\begin{array}{l}\text { DOl: 10.1159/000494867 } \\
\text { Published online: 3 November } 2018\end{array}$ & $\begin{array}{l}\odot \text { 2018 The Author(s). Published by S. Karger AG, Basel } \\
\text { www.karger.com/cpb }\end{array}$ \\
\cline { 2 - 3 }
\end{tabular} \\ Kim et al.: EGR1 Attenuates HNSCC Metastasis}

transfection of EGR1 overexpression vector by Lipofectamine 2000 for 13 days (Fig. 4A, 4C). EGR1 has been reported to promote MMP9 expression in HeLa cell [11]. However, in lymphoma, EGR1 inhibits MMP9 expression by direct promoter binding [12]. Our data showed that exogenous EGR1 overexpression inhibited mRNA and protein expression of MMP9, which is consistent with the result in lymphoma. In addition, the MDM2 promoter has two EGR1-binding consensus sequences. Therefore, we evaluated whether EGR1 inhibits transcription of MDM2 by binding directly to the consensus promoter sequences. Interestingly, MDM2 transcription was decreased significantly by direct EGR1 binding to their respective promoters. To confirm whether MMP2 or MMP9 take part in MDM2 expression, we examined the effect of specific MMP2/9 inhibitor on MDM2 protein expression. There has been no remarkable change of MDM2 expression in FaDU cell treated with MMP2/9 inhibitor. These data supports that decreased MDM2 protein expression in EGR1-overexpressed cell would be due to EGR1. As mentioned in the introduction, EGR1 is overexpressed by various extra stimuli, and its function is known to vary depending on the cell types and its environment. PMA has been reported to have different effects on EGR1 expression depending on the cell type [25]. In the present study, PMA treatment caused no remarkable change of EGR1 protein expression in FaDU cell. Next, we examined the effect of EGR1 on PMA-dependent MMP9 induction. Interestingly, exogenous EGR1 overexpression alleviated MMP9 increase with PMA treatment. These data suggests that EGR1 would take critical part to protect MMP9-dependent cancer cell invasion. A previous study showed that MDM2 promotes cancer invasion and metastasis in breast carcinoma by inducing MMP9 [13]. Furthermore, MDM2 promotes cell motility and invasion by downregulating E-cadherin expression [26]. Therefore, direct downregulation of MDM2 seem to be important molecular mechanisms of EGR1's anti-invasion function in HNSCC.

Regarding these data, we tried to evaluate whether mRNA expression of EGR1-MMP9/ MDM2 is associated with metastasis in HNSCC patients. The diverse nature of cancer progress and metastasis makes it difficult to translate in vitro studies into in vivo experiments. TCGA provides multiple types of datasets from a large patient cohort, allowing assessment of molecular mechanisms in real patient data. It is known that cancer is a complex disease with many different types of molecular mechanisms. Therefore, identifying a new subgroup connecting a certain molecular signature which represents a reasonable patient population with clinical significance would be valuable. Since we found that EGR1 expression is low in metastatic patients, we further examined if some HNSCC cancer patients present signatures of EGR1 and other associated genes, and if so, whether these patients are enriched in a certain phenotype. Interestingly, a subgroup of a large population presenting higher EGR1 expressions alongside lower MMP9 and/or MDM2 had significantly fewer metastatic patients $(P=0.025)$. EGR1 molecular mechanism was manifested among these 483 patient data, providing a basis for future research involving EGR1 function related to metastasis. In conclusion, our data shows that EGR1 downregulates MMP9 and MDM2, which are critical for HNSCC cell invasion. mRNA and protein expression pattern of HNSCC tissues supports the inhibitory effect of EGR1 on HNSCC invasion. These data may be applicable to the prognosis of HNSCC.

\section{Acknowledgements}

This work was supported by the Basic Science Research Program through a National Research Foundation (NRF) of Korea Grant funded by the Korean Government (2018R1A2B6005005), (2017R1C1B2004732).

\section{Disclosure Statement}

The authors disclose no potential conflicts of interest.

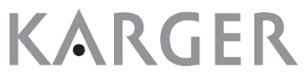




\section{Cellular Physiology Cell Physiol Biochem 2018;50:1869-1881

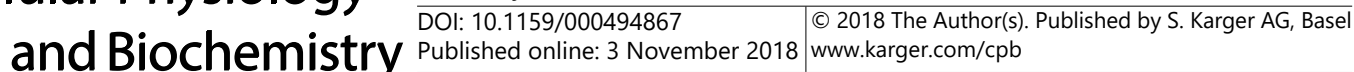 \\ Kim et al.: EGR1 Attenuates HNSCC Metastasis}

\section{References}

1 Steeg PS: Tumor metastasis: mechanistic insights and clinical challenges. Nat Med 2006;12:895-904.

- Eccles SA, Welch DR: Metastasis: recent discoveries and novel treatment strategies. Lancet 2007;369:17421757.

3 Argiris A, Karamouzis MV, Raben D, Ferris RL: Head and neck cancer. Lancet 2008;371:1695-1709.

-4 Lim RW, Varnum BC, Herschman HR: Cloning of tetradecanoyl phorbol ester-induced 'primary response' sequences and their expression in density-arrested Swiss 3T3 cells and a TPA non-proliferative variant. Oncogene 1987;1:263-270.

5 Christy BA, Lau LF, Nathans D: A gene activated in mouse 3T3 cells by serum growth factors encodes a protein with "zinc finger" sequences. Proc Natl Acad Sci U S A 1988;85:7857-7861.

6 Calogero A, Arcella A, De Gregorio G, Porcellini A, Mercola D, Liu C, Lombari V, Zani M, Giannini G, Gagliardi FM, Caruso R, Gulino A, Frati L, Ragona G: The early growth response gene EGR-1 behaves as a suppressor gene that is down-regulated independent of ARF/Mdm2 but not p53 alterations in fresh human gliomas. Clin Cancer Res 2001;7:2788-2796.

7 Levin WJ, Press MF, Gaynor RB, Sukhatme VP, Boone TC, Reissmann PT, Figlin RA, Holmes EC, Souza LM, Slamon DJ: Expression patterns of immediate early transcription factors in human non-small cell lung cancer. The Lung Cancer Study Group. Oncogene 1995;11:1261-1269.

8 Eid MA, Kumar MV, Iczkowski KA, Bostwick DG, Tindall DJ: Expression of early growth response genes in human prostate cancer. Cancer Res 1998;58:2461-2468.

-9 Zheng L, Pu J, Jiang G, Weng M, He J, Mei H, Hou X, Tong Q: Abnormal expression of early growth response 1 in gastric cancer: association with tumor invasion, metastasis and heparanase transcription. Pathol Int 2010;60:268-277.

-10 Zcharia E, Atzmon R, Nagler A, Shimoni A, Peretz T, Vlodavsky I: Inhibition of matrix metalloproteinase-2 by halofuginone is mediated by the Egr 1 transcription factor. Anticancer Drugs 2012;23:1022-1031.

11 Shin SY, Kim JH, Baker A, Lim Y, Lee YH: Transcription factor Egr-1 is essential for maximal matrix metalloproteinase-9 transcription by tumor necrosis factor alpha. Mol Cancer Res 2010;8:507-519.

12 Bouchard F, Belanger SD, Biron-Pain K, St-Pierre Y: EGR-1 activation by EGF inhibits MMP-9 expression and lymphoma growth. Blood 2010;116:759-766.

13 Chen X, Qiu J, Yang D, Lu J, Yan C, Zha X, Yin Y: MDM2 promotes invasion and metastasis in invasive ductal breast carcinoma by inducing matrix metalloproteinase-9. PLoS One 2013;8:e78794.

14 Zhang DH, Zhang LY, Liu DJ, Yang F, Zhao JZ: Expression and significance of MMP-9 and MDM2 in the oncogenesis of lung cancer in rats. Asian Pac J Trop Med 2014;7:585-588.

15 Love MI, Huber W, Anders S: Moderated estimation of fold change and dispersion for RNA-seq data with DESeq2. Genome Biol 2014;15:550.

16 Kim J, Kang HS, Lee YJ, Lee HJ, Yun J, Shin JH, Lee CW, Kwon BM, Hong SH: EGR1-dependent PTEN upregulation by 2-benzoyloxycinnamaldehyde attenuates cell invasion and EMT in colon cancer. Cancer Lett 2014;349:35-44.

17 Vinci M, Box C, Eccles SA: Three-dimensional (3D) tumor spheroid invasion assay. J Vis Exp 2015:e52686.

18 Bond GL, Hu W, Bond EE, Robins H, Lutzker SG, Arva NC, Bargonetti J, Bartel F, Taubert H, Wuerl P, Onel K, Yip L, Hwang SJ, Strong LC, Lozano G, Levine AJ: A single nucleotide polymorphism in the MDM2 promoter attenuates the p53 tumor suppressor pathway and accelerates tumor formation in humans. Cell 2004;119:591-602.

19 Fukada T, Tonks NK: The reciprocal role of Egr-1 and Sp family proteins in regulation of the PTP1B promoter in response to the p210 Bcr-Abl oncoprotein-tyrosine kinase. J Biol Chem 2001;276:2551225519.

20 Hussain H, Chong NF: Combined Overlap Extension PCR Method for Improved Site Directed Mutagenesis. Biomed Res Int 2016;2016:8041532.

21 Myung DS, Park YL, Kim N, Chung CY, Park HC, Kim JS, Cho SB, Lee WS, Lee JH, Joo YE: Expression of early growth response-1 in colorectal cancer and its relation to tumor cell proliferation and apoptosis. Oncol Rep 2014;31:788-794.

22 Kang HS, Ock J, Lee HJ, Lee YJ, Kwon BM, Hong SH: Early growth response protein 1 upregulation and nuclear translocation by 2'-benzoyloxycinnamaldehyde induces prostate cancer cell death. Cancer Lett 2013;329:217-227. 
23 Aref AR, Huang RY, Yu W, Chua KN, Sun W, Tu TY, Bai J, Sim WJ, Zervantonakis IK, Thiery JP, Kamm RD: Screening therapeutic EMT blocking agents in a three-dimensional microenvironment. Integr Biol (Camb) 2013;5:381-389.

24 Vinci M, Gowan S, Boxall F, Patterson L, Zimmermann M, Court W, Lomas C, Mendiola M, Hardisson D, Eccles SA: Advances in establishment and analysis of three-dimensional tumor spheroid-based functional assays for target validation and drug evaluation. BMC Biol 2012;10:29.

-25 You L, Jakowlew SB: Identification of early growth response gene-1 (Egr-1) as a phorbol myristate acetateinduced gene in lung cancer cells by differential mRNA display. Am J Respir Cell Mol Biol 1997;17:617-624.

-26 Yang JY, Zong CS, Xia W, Wei Y, Ali-Seyed M, Li Z, Broglio K, Berry DA, Hung MC: MDM2 promotes cell motility and invasiveness by regulating E-cadherin degradation. Mol Cell Biol 2006;26:7269-7282. 